\title{
STRENGTH AND STABILITY
}

\section{Fortaleza y estabilidad}

Paula TeiJeiro ${ }^{a, b}$

https://orcid.org/0000-0003-3906-8339

paulateijeiro@gmail.com

${ }^{a}$ IIF-SADAF, National Scientific and Technical Research Council, Buenos Aires, Argentina.

${ }^{b}$ University of Buenos Aires, Argentina.

\begin{abstract}
In this paper, I present two presumed alternative definitions of meta-validity for meta-inferences: Local and Global. I defend the latter, first, by arguing that it is not too weak with respect to meta-inference-cases, and that local meta-validity is in fact too strong with respect to types. Second, I show that although as regards metainference-schemas Local metavalidity is always stable, Global metavalidity is also stable when the language satisfies reasonable expressibility criteria (and that in fact, both concepts collapse in those cases).
\end{abstract}

Key Words: Meta-inferences; Local Metavalidity; Global Metavalidity; Substructural Logic.

\section{Resumen}

En este artículo, considero dos presuntas definiciones alternativas de validez metainferencial: local y global. Defiendo esta última, en primer lugar, argumentando que no es demasiado débil respecto de las metainferencias-caso, y que la validez local es de hecho demasiado fuerte respecto de las metainferencias-tipo. En segundo lugar, muestro que, si bien respecto de esquemas la metavalidez local es siempre estable, la metavalidez global también lo es, siempre y cuando el lenguaje satisfaga criterios razonables de expresabilidad (y que, de hecho, ambas nociones colapsan en esos casos).

Palabras clave: Metainferencias; Metavalidez local; Metavalidez global; Lógica subestructural.

\section{Introduction}

In recent years, the conversation on paradoxes has made an interesting ascent from the object level to the meta-level. Quite frequently, the logical alternatives being discussed are not characterized in terms 
of the inferences they validate (because, for instance, they coincide with classical logic or with the empty set). Instead, they are framed in terms of meta-inferences, which can be understood as relations, not between sentences, but between inferences.

To put it more formally:

Definition (Inference-case) An inference-case expressed in a language $\mathcal{L}$ is a pair $\langle\Gamma, \Delta\rangle$ of sets of $\mathcal{L}$-formulas.

Definition (Metainference-case) A metainference-case expressed in a language $\mathcal{L}$ is a pair $\langle\Sigma, \sigma\rangle$, where $\Sigma$ is a set of inference-cases and $\sigma$ is an inference-case in $\mathcal{L}^{1}$.

And the corresponding generalized concepts:

Definition ((Meta)Inference-type) A (meta)inference-type is a set of (meta)inference-cases.

I will omit the specification type/case when it does not matter. Given then that interesting theoretical action might take place at this level, it seems important to incorporate meta-inferences into our understanding of what logic is, which we can thus characterize as follows:

Definition (Logic) A logic is a set of inference-cases and metainference-cases.

I stop at meta-inferences, and do not define the concept all the way up, because it is here that we already face the issue that I want to settle. A consequence of the fact that the meta-inferential description of logic has generally been done in proof-theoretic terms (by means of sequent calculi), and consequently, model-theoretic considerations have been left a bit unattended. With respect to inferences, we do know how to use valuations to characterize logics:

Definition (Valuation) A valuation for a language $\mathcal{L}$ is a function $v$ from the set of $\mathcal{L}$-formulas to some set of values $\tau$.

We just pair some set $\mathcal{M}$ of valuations, together with a way

\footnotetext{
${ }^{1}$ To keep the symmetry, meta-inferences should also be relations between sets, but I will leave that aside here because it does not affect the point at issue.
} 
of determining a consequence relation $\vDash$. If we take for instance Boolean valuations, we can get the set of inferences obeying classical consequence, i.e, preserving value 1 , which constitute classical logic (CL). Or if we take the set of strong Kleene valuations, we can get either the inferences obeying paracomplete consequence, i.e. preserving value $\{1\}$, which constitute Strong Kleene logic (K3); or those obeying paraconsistent consequence, i.e. preserving values $\{1,1 / 2\}$, which yield the logic of paradox (LP); or those obeying strict-tolerant consequence, i.e., cannot have value 1 in the premises and 0 in the conclusion, which yield strict-tolerant logic $(\mathbf{S T})$.

The problem is then how to characterize the metainferential part of these and other logics. The at least prima facie most natural candidate for a metavalidity relation is the following global concept:

Definition (Global Metavalidity) A metainference-case is globally valid iff the conclusion is valid when the premises are.

Nevertheless, some authors like Ripley (2018), Dicher and Paoli (2018) and Barrio et al. (2020) suggested considering also or instead of this, a local one, which is less common (although not unheard of ${ }^{2}$ ):

Definition (Local Metavalidity) Say that a valuation confirms an inference when it is not a countermodel for it. A metainference-case is locally valid iff every valuation that confirms the premises, confirms the conclusion.

Why would anyone prefer this stranger concept? After all, the confirmation relation does not seem to capture any natural pre-theoretical concept. A possible route to justify it could be uniformity: given that we are not requiring inferences to preserve tautologousness but truth, we should not expect meta-inferences to preserve validity but confirmation. Nevertheless, the analogy does not quite work: while truth is important for the role sentences can have in a variety of contexts, confirmation does not seem to be useful in the same way. Learning that the intended model confirms some argument does not generate any kind of justification of the correctness of the argument, while learning that it makes some sentence true gives us, for instance, justification to believe it, assert it, etc. ${ }^{3}$

\footnotetext{
${ }^{2}$ It appears for instance in Humberstone (1996), which I will mention in the next section.

${ }^{3}$ Local metavalidity amounts to countermodel-preservation from conclusion
} ANÁLISIS FILOSÓFICO 4I(2) - (noviembre 202I) 
Despite that, some of these authors still think Global metavalidity is the inferior option, on account of two charges. In the next section, I tackle the presumed weakness of the concept whereas the third section presents the perhaps more delicate issue of its instability. I will claim that both these worries are ill-founded.

The languages I will be working with are all propositional, and notation is very simple; I will use, as customary, ' $\Gamma \Rightarrow \Delta^{\prime}$ ' as a metatheoretical name for inferences, and will graphically represent meta-inferences as follows:

$$
\frac{\Gamma 1 \Rightarrow \Delta_{1} \ldots \Gamma_{\mathrm{n}} \Rightarrow \Delta_{\mathrm{n}}}{\Gamma \Rightarrow \Delta}
$$

\section{Weakness}

Dicher and Paoli (2018) start their paper by defending two propositions. The first one is that we should be more concerned with metainference-cases than with types, especially when working within specific theories. But this does not imply their second proposition, which is that Global metavalidity is too weak.

So, let us first grant for a moment that metainference-cases are the key concept. It is indeed true that for them, Global metavalidity is a strictly weaker concept than Local metavalidity. The other direction fails because there are metainference-cases with invalid premises, and it is the existence of these vacuous examples what bothers Dicher and Paoli:

(...) [global] metainferential validity is too weak. Applied to ST, it would vindicate, e.g., every one-premiss metainference ( $\left.\{\mathrm{S}\} ; \mathrm{S}^{\prime}\right)$ such that $\mathbf{S}$ is an ST-invalid sequent and $\mathrm{S}^{\prime}$ is arbitrary. It makes little sense to pursue an inventory of such valid metainferences. (Dicher \& Paoli, 2018)

There is of course nothing peculiar to ST about this; the following case, labeled (1), is globally valid in any logic:

to premises, and countermodelling is indeed a relevant relation. But there is a disanalogy between this and falsity preservation from conclusion to premises in inferences, which is that truth and falsity are complements of each other. What matters is preservation of invalidity from conclusion to premises, which is Global metavalidity's contrapositive. 


$$
\stackrel{\Rightarrow p}{\Rightarrow q}
$$

This obviously does not mean that every metainference-case is globally valid, so triviality cannot be the reason why the pursuit of such an inventory makes so little sense. So why should (1) be considered invalid?

First, it is true that there is no "connection" between premise and conclusion, but the same happens in most logics with, for instance, inferences from $p \wedge \neg p$ to $q$. It represents no danger to the theory to vindicate those cases, given that even though it licenses the passage from $\Rightarrow p$ to $\Rightarrow q$, or from $p \wedge \neg p$ to $q$, we will not be in the position to assert $\Rightarrow p$ or $p \wedge \neg p{ }^{4}$

A similar way to plead against the metavalidity of (1) is to claim (as an anonymous referee suggested) that Local metavalidity is more fitted for an entailment-like reading of the metainferential relation, whereas the Global concept would correspond better with a conditionallike reading. The reason would be precisely that the mere falsity of " $p$ is valid" is enough to globally validate (1). The problem is that this falsity is not a material, but a logical one: logical validity is a formal operator, and the reason why $p$ is not valid has to do with its atomic form, not with its content5. So this fact alone is not enough to distinguish between conditionals and entailments.

But not only there seems to be no reason to consider cases like that in (1) to be invalid metainferences, but actually there are some in favor of considering it valid. Undoubtedly, a proper definition has to work not only for metainference-cases, but also for general types, regardless of which we think should be our main focus of interest. But the concept of Local metavalidity works poorly when applied to types. As an example, take the rule of Uniform Substitution (US), which states that if an inference belongs to a certain logic, all inferences obtained by substituting its propositional variables by any formula have to belong in it also. (US) is a closure condition, and is very commonly thought to be a necessary requirement for any logic (although not a requisite for any theory which is not pure). And (US) is only globally valid, not locally so, given that (1) is in fact an instance of the type. ${ }^{6}$

\footnotetext{
${ }^{4}$ Relevantist concerns are of course perfectly legitimate, but that does not seem to be the place where these authors are coming from.

5 If $p$ is not necessarily logically atomic, then it is a metavariable rather than a variable. We look at this case in the next section.

${ }^{6}$ The problem here concerns Dicher and Paoli's specific purpose in their paper,
} 
I think this is enough to rule out Local metavalidity as the best choice when it comes to consequence relations. There is a different issue related to vacuously globally valid metainferences, however, which I consider in the next section.

\section{Instability}

The reason why the distinction between Local and Global metavalidity is often overlooked is that in logic we generally tend to consider, not particular instances, or metainference-types in general, but a special subset of them:

Definition (Schematic (Meta)inference) A schematic (meta) inference is a type where all the members can be obtained from a single one by uniformly substituting some propositional variable $p$ by some formula $A$.

And for them, Local and Global metavalidity usually coincide. For example, in $\mathbf{C L}$, the schema to the left is not globally valid, since it has as an instance, besides (1), also the substitution to its right:

$$
\begin{array}{ll}
\Rightarrow A & \Rightarrow p \vee \neg p \\
\Rightarrow B & \Rightarrow q
\end{array}
$$

In schemas, the entailment represented by Global metavalidity is not merely necessary, as we said in the previous section, but it regains formality. For Ripley (2018), the interest lies more in schemas than in particular instances, and the issue he has with Global metavalidity does not merely rely on the fact that there are vacuous cases. Schemas are important because they serve as rules of inference. It is because of this that we have better accounts of schematic metainferences in terms of proof theory than model-theoretically. In sequent calculi, there are two metalevel consequences which are often considered:

which is to show that ST's classicality claims are overstated, and that the logic that really captures ST's essence is the paraconsistent LP. For that, they set up a caseby-case translation from metainferences to inferences, which, for instance, turns (1) into $p \Rightarrow q$. Given that this is an LP-invalid sequent, Global metavalidity would not determine an LP kind of logic. This, of course, depends on the mapping. If we translate (1) into $\mathrm{T} \rightarrow p \Rightarrow \mathrm{T} \rightarrow q$, which is an LP-valid sequent, the point can still be made. Another option would be to make a schema-to-schema comparison, which is what Barrio et al. (2015) do. 
Definition (Derivability) A metainference-type is derivable in a system S iff, for all its members, the conclusion can be proved in the system which results from adding the premises to $\mathrm{S}$.

Definition (Admissibility) A metainference-type is admissible in a system $S$ iff, for all its members, the conclusion can be proved whenever the premises can be proved.

Extrapolating Pogorzelski's terminology to the metalevel-as Humberstone (2011) does-we call the logics where schematic admissible and derivable rules coincide structurally complete. Some logics have structurally complete calculi, like $\mathbf{C L}$, and some do not, like intuitionistic logic. CL also has non-structurally complete presentations, like the case without explicit mention of the Cut rule.

A proof system theorizes about the concept of derivability, but in general nothing too significant hinges on the distinction between these two concepts. The reason is that making admissible rules explicit does not affect the set of provable sequents, and thus a gap between derivability and admissibility does not matter when theorizing about the object level. The only thing that counts is for the system to be sound and complete with respect to the object language consequence relation it is trying to capture. Presenting a system with some rules left admissible is a convenient way to simplify presentations and/or proofs.

In ideal circumstances, the link between Global metavalidity and Admissibility is pretty straightforward:

Fact 3.1 If $S$ is sound and complete with respect to the logic determined by $\langle\mathcal{M}, \vDash\rangle$, then Global metavalidity and Admissibility coincide.

Nevertheless, Ripley (2018) points out that merely admissible rules may not be valid in the case of incomplete systems. For instance, take the Bare Calculus (BC), a system without any rules for the connectives. This system is very incomplete with respect to $\mathbf{C L}$, it does not even prove one instance of $A \wedge B \Rightarrow A$. At the same time, the rule of Swap is admissible, since the only provable sequents are those where premises and conclusions share formulas:

$$
\frac{\Gamma \Rightarrow \Delta}{\Delta \Rightarrow \Gamma} \quad \text { (Swap) }
$$


But Swap is clearly invalid, given that, following the same example, $A \Rightarrow A \wedge B$ is not in $\mathbf{C L}$. This particular case is not worrisome, because we indeed have complete calculi for CL. But that is not always the case. For Ripley, the goal is to capture a relation which represents material validity for natural language, and includes things like "Something being green implies that it is not yellow". This is a theory which has not yet been obtained, and probably can only be approximated. The problem then is how to guarantee that the rules we take to define some piece of vocabulary will continue to hold as we enrich the theory.

If we have the whole vocabulary in the language from the beginning, restricting available interpretations will not affect the derivability of the schemas, because they grant inferential permissions, which cannot be taken away: if there is a derivation in some system, then adding new rules will not make this derivation impossible. At most, you can allow too much.

Admissible metainferences, nevertheless, need not be stable: strengthening the system increases the set of provable sequents, and rules which were admissible merely because its premises were unprovable may turn inadmissible because those rules were validated. This is why, according to Ripley, we should only consider derivable rules as meaning-conferring.

The same problem affects Global metavalidity, if we consider it in tandem with another consequence relation for which it is not complete. Restricting models can make premises valid, so if we are adding new rules to interpret some vocabulary, merely checking that they are globally valid might not be enough.

Although it would be nice to have Local metavalidity coincide with Derivability, in the same natural way Global metavalidity and admissibility do, they do not, as Humberstone (1996) showed. There, he defines another concept of "true" Global metavalidity:

Definition (Absolute Global metavalidity) A rule is absolutely globally valid with respect to some set $\mathbb{V}$ of sets of valuations (its global range) and a consequence relation for them iff it preserves $\mathcal{M}$-validity for every $\mathcal{M} \in \mathbb{V}$.

And then he proves that, for structural logics, underivable rules are not globally $\mathcal{M}$-valid with respect to some $\mathcal{M}$ that the rest of the system rules do preserve. Thus, Local metavalidity does not get the prestige of being a guide to Derivability.

Nevertheless, Local validity does enjoy this nice property of 
being stable. It is because of it that Ripley argues we should check for Local instead of Global metavalidity when incorporating new rules: if a metainference is locally valid with respect to some class of models, it will be valid for any subset of it. In the next section we will see how this sort of caution is only necessary for fairly poor languages.

\section{The Path to Stability}

The gap between Admissibility and Derivability, as the one between Global metavalidity and Absolutely Global metavalidity, is sometimes not that easy to bridge. Having a structurally complete calculus -even when possible- might not be easy. On the contrary, closing the gap between Local and Global Metavalidity is not such a difficult task.

On the one hand, the two following semantic properties are at least sufficient:

Definition (Truth-functionality) A logic is truth-functional with respect to a set of valuations $v$ with codomain $\tau$ iff for every n-ary propositional operator symbol $\odot$ there is a function $f: \tau^{\mathrm{n}} \rightarrow \tau$ such that $v(\odot(A 1, \ldots, A \mathrm{n}))=f(v(A 1), \ldots, v(A \mathrm{n}))$.

Definition (Truth Constant-completeness) A logic is truthconstant complete iff for every value $\mathbf{a} \in \tau$ there is a formula Aa which takes value $\mathbf{a}$ in every valuation

Fact 4.1 If a logic is truth-constant complete and truthfunctional, Global metavalidity implies Local metavalidity for schematic metainferences.

Proof: Assume $\mathrm{R}$ is not locally valid. Then, there is a member of $R$ such that there is a $v$ which confirms each $\Gamma_{i} \Rightarrow \Delta$ but does not confirm $\Gamma \Rightarrow \Delta$. Take the substitutional instance which assigns $A$ a to every propositional variable such that $v(p)=\mathbf{a}$. Every valuation confirms the premises and none the conclusion of this instance, which makes it globally invalid. Hence, $R$ is not globally valid.

Thus, the stability which was Local Metavalidity's main asset will also be available for Global metavalidity in these cases: restricting models may introduce more valid arguments, but this will not affect schemas. This result has two main limitations: (a) some logics are not truth-functional (b) some logics are not truth-constant complete. 
Starting with (b), some logics cannot be truth-constant complete without infinitary syntax, given that they are truth-functional only with respect to sets $\tau$ with uncountably infinite values. In some cases, though, it might suffice to incorporate some countable subset of those to obtain the equivalence between Global and Local metavalidity. Nevertheless, that will not be the case in general.

Regarding (a), on the other hand, I do not have a general answer, but I want to illustrate how at least in two cases, not having truthfunctionality will not work against Global metavalidity. In the first and easier one, we do not get the collapse, but it does not matter because the local concept is plainly wrong. The case I am referring to is that of a modal language, where we typically want the following inference to be valid:

$$
\begin{aligned}
& \Rightarrow A \\
& \Rightarrow \square A
\end{aligned}
$$

which is also typically locally invalid.

The second case to look at is S-valuationism, and what happens here is that we get the collapse result despite not having truthfunctionality. S-valuationism is a family of logics characterized by models which are spaces of classical points (see for instance Fine, 1975; Jaśkowsky, 1969; Keefe, 2000). Briefly, the general idea is that sentences in those models are evaluated as either supertrue - if they have value 1 in every point in the space- or subfalse -if they have value 0 in some point. Logical consequence can be defined as preservation of supertruth -which gives you the logic called Supervaluationism - or subtruth which gives you Subvalutionism.

One of S-valuationism's most salient features is that it is noncompositional: for instance $A \vee \neg A$ is supertrue in every model, even though in some, for some $A$, neither $A$ nor $\neg A$ are. This allows for more classicality than other paracomplete or paraconsistent alternatives: in a single-premise framework (For-Set), Subvaluationism has the same valid arguments as $\mathbf{C L}$, while Supervaluationism has them when considered in the more standard single-conclusion one (Set-For). Nevertheless, regarding metainferences, the classicality depends on whether we consider the consequence to be Global or Local.

A space confirms a For-Set argument according to Subvaluationism if and only if either the premise is superfalse or some conclusion is subtrue, and it confirms a Set-For argument according to Supervaluationism if and only if either some premise is subfalse 
or the conclusion is supertrue. Then the following versions of $\vee \mathrm{L}$ and $\wedge R$ have instances (those below them) which have respectively local supervaluational and subvaluational countermodels:

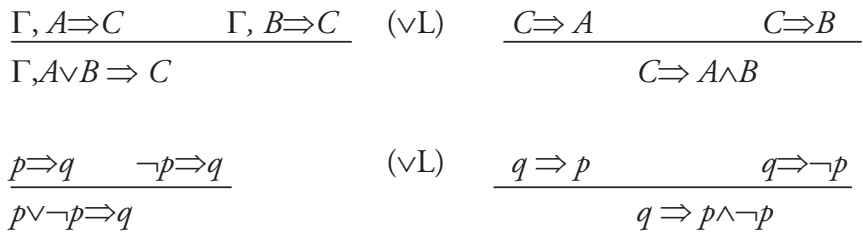

Any space in which both $p$ and $\neg p$ are subfalse (true) will confirm the premises of $\vee \mathrm{L}(\wedge \mathrm{R})$ according to a supervaluationist (subvaluationist) standard, but some of those spaces will make $q$ superfalse (subtrue), and thus will be counterexamples to the conclusion. Still, these metainferences are globally valid.

In the case of S-valuationism, there is no truth-value constant to add, because semantic values in a model are not the codomain of some valuation function. Nevertheless, there are other ways to make Global metavalidity stricter. For instance, if we take the more usual enrichments of the language, which consist in adding a possibility operator $\diamond A$ (meaning $A$ is at least subtrue), or a necessity operator $\square A$ (meaning $A$ is supertrue), then we get the following globally invalid instances:

$\frac{p \Rightarrow \square p \vee \square \neg p \quad \neg p \Rightarrow \square p \vee \square \neg p}{p \vee \neg p \Rightarrow \square p \vee \square \neg p} \quad(\vee \mathrm{L}) \quad \frac{\diamond p \wedge \diamond \neg p \Rightarrow p \quad \nabla p \wedge \diamond \neg p \Rightarrow \neg p}{\diamond p \wedge \diamond \neg p \Rightarrow p \wedge \neg p} \quad(\wedge \mathrm{R})$

Another option would be to incorporate something which in a sense emulates a truth-constant. Even though the language has the resources to represent a constant for supertruth -any tautology- and a constant for superfalsity -any contradiction- there is no formula which has the value subtrue-and-subfalse in every space. If $\lambda$ is such a formula, then these are the corresponding globally invalid instances: ${ }^{7}$

$$
\frac{\lambda \Rightarrow \quad p \quad \neg \lambda \Rightarrow p}{\lambda \vee \neg \lambda \Rightarrow p} \quad(\vee \mathrm{L}) \quad \frac{p \Rightarrow \lambda \quad p \Rightarrow \neg \lambda}{p \Rightarrow \lambda \wedge \neg \lambda} \quad(\wedge \mathrm{R})
$$

\footnotetext{
${ }^{7}$ As a consequence of taking this route, we exclude spaces made up only of one point, and thus classical models will no longer be a subset of the s-valuationist ones.
} 
A third option is to change the underlying framework. With multiple conclusions, $\vee \mathrm{L}$ is globally invalid according to supervaluationism, and with multiple premises, $\wedge \mathrm{R}$ is globally invalid according to subvaluationism:

$\frac{p \Rightarrow p \quad \neg p \Rightarrow \neg p}{p \vee \neg p \Rightarrow p, \neg p}$

$$
\frac{p \Rightarrow p \quad \neg p \Rightarrow \neg p}{p, \neg p \Rightarrow p \wedge \neg p}
$$

In this case, the enrichment is not related with the vocabulary of the object language, but with that of the metalanguage, allowing commas to appear respectively on the right or on the left.

\section{Conclusion}

When it comes to evaluating the strength of both concepts, we have seen on the one hand, that choosing to focus on metainferencecases does not turn the scale against Global metavalidity, and on the other hand, that when we consider the full class of metainference-types, Local metavalidity turns out to be too strong.

With respect to the problem of stability which affects schemas, I argued that the concept of Local metavalidity works more as a technical tool than as a proper definition of consequence, in that it allows identifying those rules which are stable across expansions of the language. But the extent to which this tool is necessary is restricted to those occasions where the object language is not rich enough to, in a sense, reflect the model theory.

The goal of this paper has been mostly to clarify the distinction between these two concepts. There are, I think, topics on metainferences which are more philosophically pressing than the one discussed here, such as whether it makes sense to accept one logic ruling inferences and a different one ruling metainferences, or whether validity is itself a classical notion (as most non-classical theorists believe), or, on the contrary, it is as infected by indeterminacy as truth is. In order to tackle those, it is important not to get caught in false controversies.

\section{References}

Barrio, E., Rosenblatt, L., \& Tajer, D. (2015). The logics of strict-tolerant logic. Journal of Philosophical Logic, 44(5), 551-571. https://doi. org/10.1007/s10992-014-9342-6

Barrio, E., Pailos, F., \& Szmuc, D. (2020). Hierarchies of para-consistency 
and classicality. Journal of Philosophical Logic, 49(1), 93-120. https://doi.org/10.1007/s10992-019-09513-z

Dicher, B., \& Paoli, F. (2018). ST, LP, and tolerant metainferences. In C. Başkent \& T. M. Ferguson (Eds.), Graham Priest on dialetheism and paraconsistency (pp. 383-407). Springer. https://doi. org/10.1007/978-3-030-25365-3_18

Fine, K. (1975). Vagueness, truth and logic. Synthese, 30(3/4), 265-300. http://www.jstor.org/stable/20115033

Humberstone, L. (1996). Valuational semantics of rule derivability. Journal of Philosophical Logic, 25(5), 451-461.

Humberstone, L. (2011). The connectives. The MIT Press.

Jaśkowski, S. (1969). Propositional Calculus for contradictory deductive systems (Communicated at the Meeting of March 19, 1948). Studia Logica: An International Journal for Symbolic Logic, 24, 143-160. http://www.jstor.org/stable/20014534 (Originally published in 1948 in Polish in Studia Scientarium Torunensis, Sec. A 11: 55-77).

Keefe, R. (2000). Theories of vagueness. Cambridge University Press.

Ripley, D. (2018). Uncut. Manuscript.

Received $20^{\text {th }}$ September 2018; revised 18 ${ }^{\text {th }}$ March 2019; accepted $21^{\text {st }}$ March 2019. 